\title{
Real-Space Mapping of Exciton Wave Functions in a Quantum Dot with Near-Field Optical Imaging Spectroscopy
}

\author{
T. SAIKI ${ }^{a, b, *}$ K. Matsuda ${ }^{b, c}$ S. Nomura ${ }^{d, e}$, M. Mihara ${ }^{e}$, \\ Y. AOYAGI $I^{e, f}$, S. NAIR ${ }^{g}$ AND T. TAKAGAhaRA ${ }^{h}$ \\ ${ }^{a}$ Department of Electronics and Electrical Engineering, Keio University \\ 3-14-1 Hiyoshi, Kohoku, Yokohama, Kanagawa 223-8522, Japan \\ ${ }^{b}$ Kanagawa Academy of Science and Technology, Japan \\ ${ }^{c}$ PRESTO Japan Science and Technology Corporation (JST), Japan \\ ${ }^{d}$ University of Tsukuba, Japan \\ ${ }^{e}$ Institute of Physical and Chemical Research (RIKEN), Japan \\ ${ }^{f}$ Tokyo Institute of Technology, Japan \\ ${ }^{g}$ University of Toronto, Canada \\ ${ }^{h}$ Kyoto Institute of Technology, Japan
}

\begin{abstract}
An exciton confined within a quantum dot acts as a two-level quantum system, and is one of the most promising candidates for quantum computing and quantum information processing. The real-space optical probing of the quantum eigenstates in a single quantum dot and coupled quantum dots should be developed toward the realization of quantum photonic devices, where their wave functions are dynamically controlled by coherent optical techniques. Here we apply near-field photoluminescence imaging spectroscopy with a high spatial resolution of $30 \mathrm{~nm}$ to map out the centre-of-mass wave function of an exciton confined in a GaAs quantum dot. The spatial profile of the exciton emission, which reflects the shape of a monolayer-high island, differs from that of biexciton emission, due to different distributions of the polarization field for the exciton and biexciton recombinations.
\end{abstract}

PACS numbers: 78.55.Cr, 78.67.Hc

${ }^{*}$ corresponding author, 


\section{Introduction}

An exciton in a naturally formed quantum dot (QD) (a monolayer-high island formed in a narrow quantum well (QW) [1-6]), which exhibits long duration of coherence at low temperature [5] and a large dipole moment [6], provides us with light-controllable quantum bits. These characteristics of an exciton quantum system in a QD have been measured by making full use of precise optical spectroscopy in both the frequency and time domains with a subwavelength spatial resolution to address a single QD. However, to improve such parameters as coherence time and dipole moment, accurate information on the wave function for individual QDs is of great importance. In addition, in the study of coupled-QDs systems as multi-quantum bits, in which it is difficult to predict the exact wave function within theoretical frameworks, an optical spectroscopic technique for probing the wave function itself should be developed. Using optical microscopy to achieve a spatial resolution in the range of $10-30 \mathrm{~nm}$, which is smaller than the typical size of QDs, local probing allows direct mapping of the real space distribution of the quantum eigenstate (wave function) within a $\mathrm{QD}$, as predicted by theoretical studies $[7,8]$.

Here we report photoluminescence (PL) imaging spectroscopy of a GaAs QD, by near-field scanning optical microscope (NSOM) with a spatial resolution of $30 \mathrm{~nm}$. This unprecedented high spatial resolution relative to the size of the QD, which is drastically improved in comparison with a typical spatial resolution of $100-200 \mathrm{~nm}$ in previous measurements [9-12], is expected to permit a real-space mapping of the centre-of-mass wave function of an exciton confined in the QD. For the target of the imaging spectroscopy, we prepared a $5 \mathrm{~nm}$ thick GaAs QW, sandwiched between layers of $\mathrm{Al}(\mathrm{Ga}) \mathrm{As}$ grown by molecular-beam epitaxy. Two-minute interruptions of the growth process at both interfaces promote the formation of natural QDs [1-6]. The narrow GaAs QW layer was covered with a thin barrier and a cap layer of totally $20 \mathrm{~nm}$, allows the near-field tip to be close enough to the emission source. The critical element in the high-resolution NSOM measurement was an optical fibre probe, which was fabricated by chemical etching with a hydrofluoric acid solution [13,14]. The optical fibre probe with a $20 \mathrm{~nm}$ clear aperture was prepared. A spatial resolution as high as $30 \mathrm{~nm}$ has been demonstrated by low temperature PL imaging of self-assembled InAs QDs with a $30 \mathrm{~nm}$ aperture probe [13].

\section{Experimental result}

We show near-field PL spectra of a single QD at $9 \mathrm{~K}$ at excitation powers ranging from 0.17 to $3.8 \mu \mathrm{W}$ in Fig. 1a. The emission lines (denoted by $X$ and $X^{*}$ ) at 1.6088 and $1.6104 \mathrm{eV}$ are observed. With an increase in excitation power, another emission line appears at $1.6057 \mathrm{eV}$ (denoted by $X X$ ). In order to clarify the origin of these emissions, we show excitation power dependence of PL intensi- 

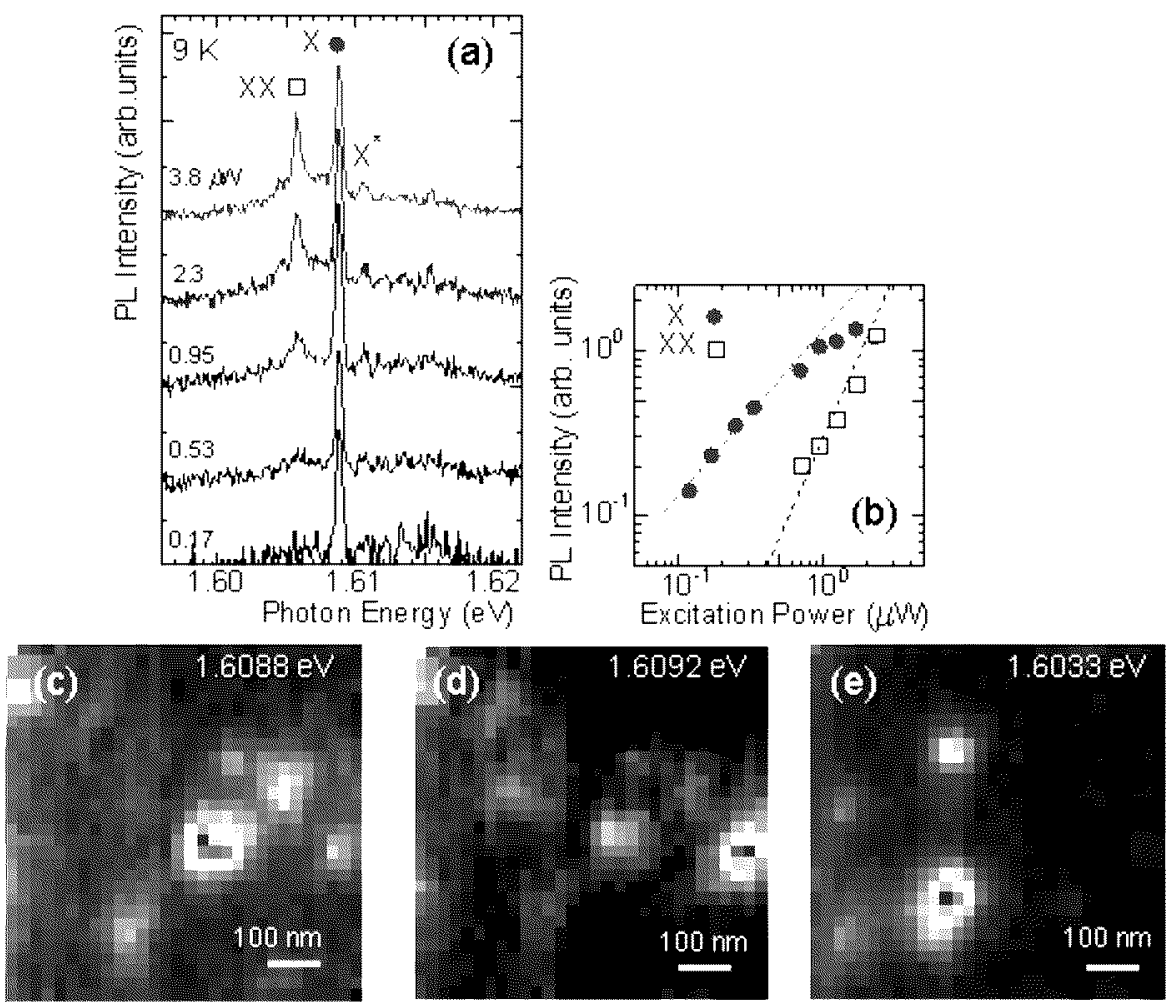

Fig. 1. (a) Near-field PL spectra of a single QD at $9 \mathrm{~K}$. The sample was non-resonantly excited with $\mathrm{He}-\mathrm{Ne}$ laser light $(\lambda=633 \mathrm{~nm})$ and the resultant $\mathrm{PL}$ signal was collected via the same aperture. The PL peaks at $1.6088,1.6057$, and $1.6104 \mathrm{eV}$ are denoted by $X, X X$ and $X^{*}$. (b) Excitation power dependence of PL intensities of the $X$ and the $X X$ lines. The red (blue) dotted line corresponds to the gradient associated with linear (quadratic) power dependence. (c)-(e) Near-field PL images obtained by mapping the intensity of the $X$ lines in the same scanning area $(1000 \times 1000 \mathrm{~nm})$.

ties in Fig. 1b. The $X$ line can be identified as an emission from a single-exciton state by its linear increase in emission intensity and its saturation behaviour. The quadratic dependence of the $X X$ emission with excitation power indicates that $X X$ is an emission from a biexciton state. This identification of the $X X$ line is also supported by the correlation energy of $3.1 \mathrm{meV}$, which agrees well with the values reported previously $[3,15]$. The $X^{*}$ emission line can be attributed to the radiative recombination of the exciton excited state by considering its energy position (higher energy side of the single exciton emission by about $1.6 \mathrm{meV}$ ) [16]. Figures $1 c-e$ show low-magnification PL maps for the intensity of $X$ emissions with three different energies in the same scanning area. These exciton PL maps show emission profiles of individual QDs, which are seen as bright spots. 
The high-magnification PL images in Fig. 2 were obtained by mapping the PL intensity with respect to the $X((\mathrm{a}),(\mathrm{c})$, and (e)) and the $X X((\mathrm{~b}),(\mathrm{d})$, and (f)) lines of three different QDs. The exciton PL images in Fig. 2 ((a), (c), and (e)) show an elongation along the $[-110]$ crystal axis. The image sizes are larger than the PL collection spot diameter, i.e. the spatial resolution of the NSOM [13]. The elongation along the $[-110]$ axis due to the anisotropy of the monolayer-high island is consistent with previous observations with a scanning tunneling microscope (STM) [2]. We also obtained elongated biexciton PL images along the [-110] crystal axis in Fig. 2 ((b), (d), and (f)) and found a clear difference in the spatial distribution between the exciton and biexciton emission. Here the significant point is that the PL image sizes of biexcitons are always smaller than those of excitons.
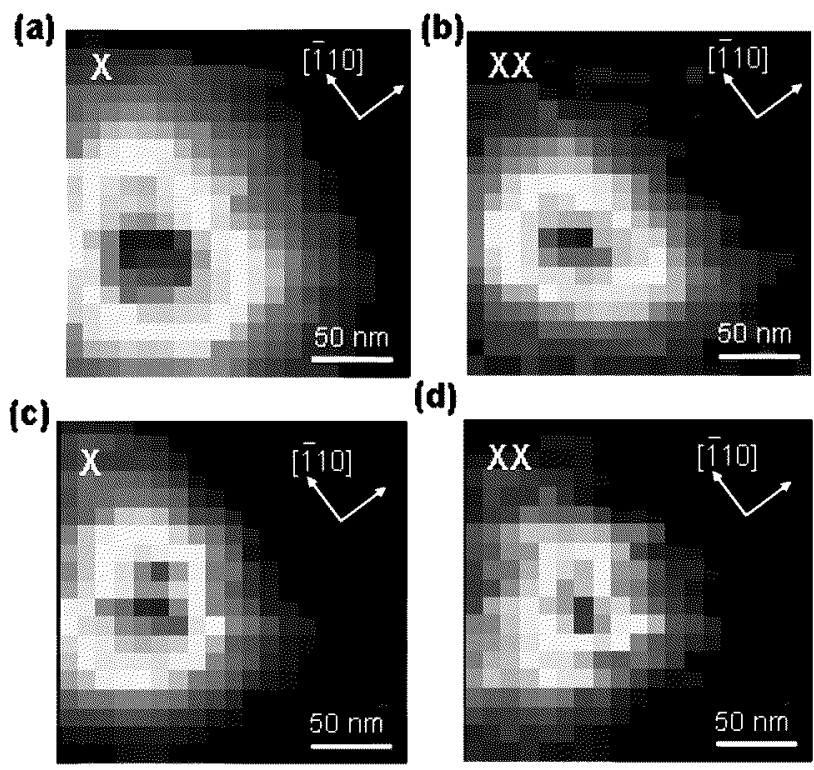

(d)
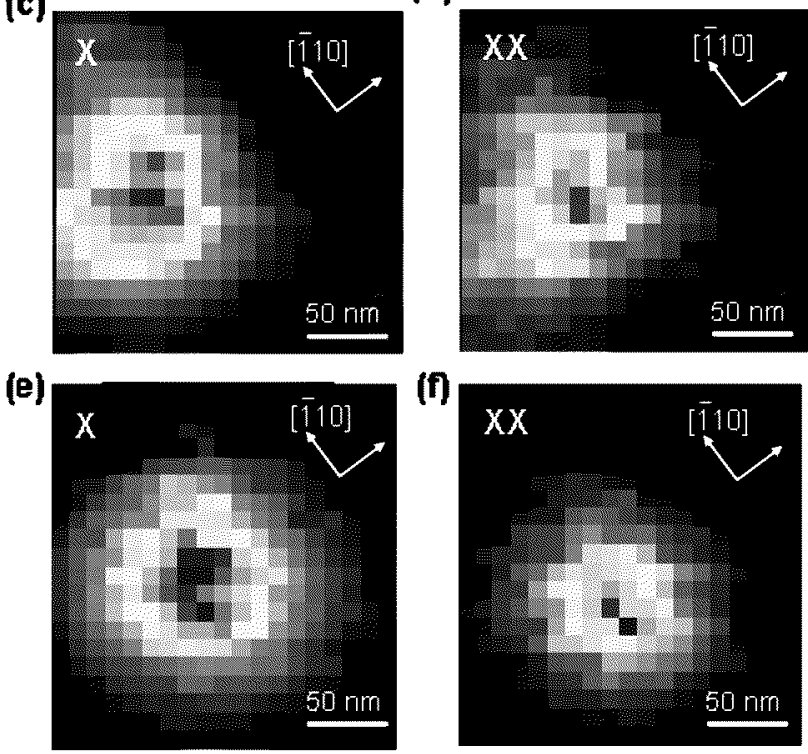

Fig. 2. (a)-(f) Series of high-resolution PL images of exciton state ((a), (c), and (e)) and biexciton state ((b), (d), and (f)) for three different QDs. Scanning area is $210 \times 210 \mathrm{~nm}$. Crystal axes along [110] and [-110] directions are indicated. PL image sizes of biexciton are always smaller than those of exciton. 


\section{Discussion and conclusion}

Figures $3 \mathrm{a}$ and $\mathrm{b}$ show the normalized cross-sectional PL intensity profiles of exciton (red) and biexciton (black) (corresponding to the profile of Figs. 2a and b) along the [110] and [-110] crystal axes. The spreads in the exciton (biexciton) images, defined as the full width at half maximum (FWHM) of each profile are $80(60) \mathrm{nm}$ and $115(80) \mathrm{nm}$ along the [110] and [-110] crystal axes, respectively. Theoretical considerations can clarify what we see in the exciton and biexciton
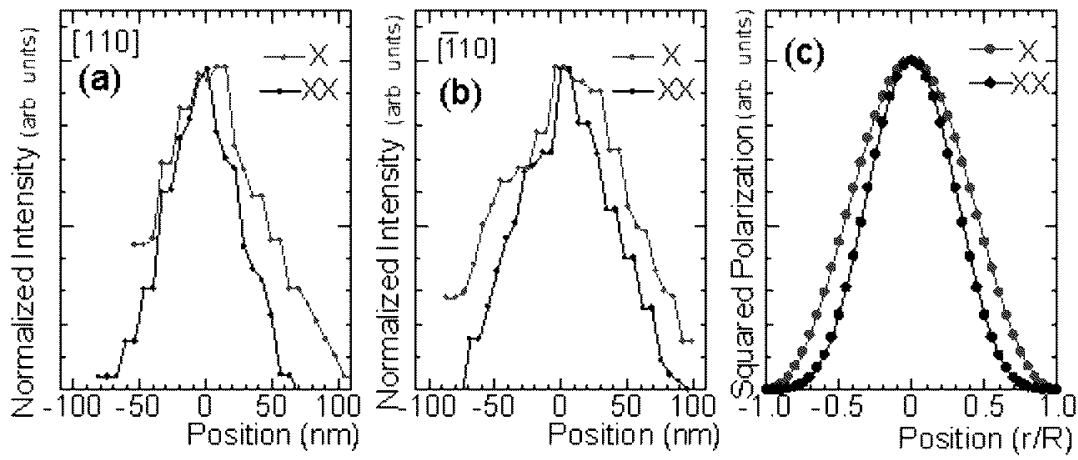

Fig. 3. (a), (b) Normalized cross-sectional intensity profiles of exciton (red) and biexciton (black) PL images corresponding to Figs. 2a and b. The image sizes of the exciton (biexciton), defined as the full width at half maximum are, $80(60) \mathrm{nm}$ and $115(80) \mathrm{nm}$ along the $[110]$ and $[-110]$ crystal axes, respectively. (c) Spatial distributions of squared polarization fields of the exciton (red) and biexciton (black) emission, which are theoretically calculated for a GaAs quantum dot (radius of $114 \mathrm{~nm}$, thickness of $5 \mathrm{~nm}$ ). The horizontal axis is normalized by the disk radius $R$.

PL images. The relevant quantity is the optical near-field around a single QD associated with an optical transition. This field can be calculated with Maxwell's equations using the polarization field of the exciton or biexciton as the source term. The observed luminescence intensity is proportional to the square of the near-field detected by the probe. In the following, however, we have calculated the emission patterns simply by the squared polarization fields without taking account of the instrumental details. The polarization fields are derived from the transition matrix element from the exciton state $(X)$ to the ground state $(0)$ and that from the biexciton state $(X X)$ to the exciton state $(X)$ as follows $[17,18]$ :

$$
\begin{aligned}
& \left\langle 0\left|p \delta\left(r-r_{s}\right)\right| X\right\rangle=-2^{1 / 2} p_{\mathrm{cv}} \varphi\left(r_{s}, r_{s}\right) \\
& \left\langle X\left|p \delta\left(r-r_{s}\right)\right| X X\right\rangle=-(3 / 2)^{1 / 2} p_{\mathrm{cv}} \sum_{r_{1}, r_{a}} \varphi\left(r_{1}, r_{a}\right) \Phi^{++}\left(r_{1}, r_{s} ; r_{a}, r_{s}\right) \\
& \quad-(1 / 6)^{1 / 2} p_{\mathrm{cv}} \sum_{r_{1}, r_{a}} \varphi\left(r_{1}, r_{a}\right) \Phi^{--}\left(r_{1}, r_{s} ; r_{a}, r_{s}\right)
\end{aligned}
$$


where $\varphi\left(r_{\mathrm{e}}, r_{\mathrm{h}}\right)$ stands for the exciton envelope function with the electron and hole coordinates denoted by $r_{\mathrm{e}}, r_{\mathrm{h}}, \Phi^{++}\left(\Phi^{--}\right)\left(r_{1}, r_{2} ; r_{a}, r_{b}\right)$ represents the biexciton envelope function with electron coordinates $\left(r_{1}, r_{2}\right)$ and hole coordinates $\left(r_{a}, r_{b}\right)$ that is symmetrised (anti-symmetrised) with respect to the interchange between two electrons and between two holes, and $p_{\mathrm{cv}}$ is the transition dipole moment between the conduction band and the valence band. As seen in Eq. (1), the spatial distribution of the exciton polarization field corresponds to the centre-of-mass envelope function of a confined exciton. For the biexciton emission, the polarization field is determined by the overlap integral, which represents the spatial correlation between two excitons forming the biexciton and is expected to be more localized than the single exciton wave function.

Figure $3 \mathrm{c}$ shows the squared polarization amplitudes of the exciton (red) and biexciton (black) emission, which have been calculated for a GaAs QD with size parameters relevant to our experiments. The calculated profile of the squared polarization amplitude of the biexciton emission is narrower than that of the exciton emission. The spread of the biexciton emission normalized by that of the exciton emission (defined by the ratio of FWHM of calculated profiles in Fig. 3c) is estimated to be 0.76 , which is in good agreement with the experimental result $(0.75 \pm 0.08)$. This theoretical support and the experimental facts lead to the conclusion that the local optical probing by the near-field scanning optical microscope directly maps out the centre-of-mass wave function of an exciton confined in a monolayer-high island.

The success of the real-space mapping of the exciton wave function described above is a step toward the realization of quantum devices, because the combination of local optical probing with time-domain coherent control using ultrafast laser pulses will permit advanced manipulation of the QD wave function with direct monitoring of the shape and extent of the wave function itself. Moreover, the optical probe, with its high spatial precision of $30 \mathrm{~nm}$, provides a bridge between the measurements of nanometre-resolution electron microscopy and wavelength-resolution far-field optical spectroscopy, and could play an active role in the characterization of not only well-defined systems as demonstrated here, but also unknown material systems in diverse fields of research.

\section{Acknowledgments}

We thank M. Ohtsu and S. Mononobe for technical advice including the fabrication of optical fibre probe. This study was supported in part by the Active Nano-Characterization and Technology Project, Special Coordination Funds of the Ministry of Education, Culture, Sports, Science and Technology of the Japanese Government. 


\section{References}

[1] K. Brunner, G. Abstreiter, G. Böhm, G. Tränkle, G. Weimann, Phys. Rev. Lett. 73, 1138 (1994).

[2] D. Gammon, E.S. Snow, B.V. Shanabrook, D.S. Katzer, D. Park, Phys. Rev. Lett. 76, 3005 (1996).

[3] G. Chen, T.H. Stievater, E.T. Batteh, X. Li, D.G. Steel, D. Gammon, D.S. Katzer, D. Park, L.J. Sham, Phys. Rev. Lett. 88, 117901-1 (2002).

[4] T.H. Stievater, X. Li, D.G. Steel, D. Gammon, D.S. Katzer, D. Park, C. Piermarocchi, L.J. Sham, Phys. Rev. Lett. 87, 133603-1 (2001).

[5] N.H. Bonadeo, J. Erland, D. Gammon, D. Park, D.S. Katzer, D.G. Steel, Science 282, 1473 (1998).

[6] J.R. Guest, T.H. Stievater, X. Li, J. Cheng, D.G. Steel, D. Gammon, D.S. Katzer, D. Park, C. Ell, A. Thränhardt, G. Khitrova, H.M. Gibbs, Phys. Rev. B 65, 241310-1 (2002).

[7] G.W. Bryant, Appl. Phys. Lett. 72, 768 (1998).

[8] C.D. Simserides, U. Hohenester, G. Goldoni, E. Molinari, Phys. Rev. B 62, 13657 (2000).

[9] H.F. Hess, E. Betzig, T.D. Harris, L.N. Pfeiffer, K.W. West, Science 264, 1740 (1994).

[10] F. Intonti, V. Emiliani, Ch. Lienau, T. Elsaesser, V. Savona, E. Runge, R. Zimmermann, R. Nötzel, K.H. Ploog, Phys. Rev. Lett. 87, 076801-1 (2001).

[11] J.R. Guest, T.H. Stievater, Gang. Chen, E.A. Tabak, B.G. Orr, D.G. Steel, D. Gammon, D.S. Katzer, Science 293, 2224 (2001).

[12] K. Matsuda, T. Saiki, H. Saito, K. Nishi, Appl. Phys. Lett. 76, 73 (2000).

[13] K. Matsuda, T. Saiki, S. Nomura, M. Mihara, Y. Aoyagi, Appl. Phys. Lett. 81, 2291 (2002).

[14] T. Saiki, K. Matsuda, Appl. Phys. Lett. 74, 2773 (1999).

[15] Q. Wu, R.D. Grober, D. Gammon, D.S. Katzer, Phys. Rev. B 62, 13022 (2000).

[16] D. Gammon, E.S. Snow, D.S. Katzer, Appl. Phys. Lett. 67, 2391 (1995).

[17] S.V. Nair, T. Takagahara, Phys. Rev. B 55, 5153 (1997).

[18] D.A. Kleinman, Phys. Rev. B 28, 871 (1983). 